\title{
Postprandial effects on twitch transdiaphragmatic pressure
}

\author{
W.D-C. Man*, Y-M. Luo*, N. Mustfa*, G.F. Rafferty*, J-C. Glerant*, M.I. Polkey\#, J. Moxham*
}

Postprandial effects on twitch transdiaphragmatic pressure. W.D-C. Man, Y-M. Luo, N. Mustfa, G.F. Rafferty, J-C. Glerant, M.I. Polkey, J. Moxham. (C)ERS Journals Ltd 2002.

ABSTRACT: Twitch transdiaphragmatic pressure (Pdi,tw), measured following magnetic stimulation of the phrenic nerves, is used to assess diaphragm strength, contractility and fatigue. Although the effects of posture, lung volume and potentiation on $P_{\mathrm{di}, \mathrm{tw}}$ are well described, it is not known whether the degree of gastric filling affects the measurement.

$P_{\mathrm{di}, \mathrm{tw}}$ was recorded in seven healthy volunteers on two occasions with antero-lateral magnetic stimulation of the phrenic nerves. On the first occasion, the subjects had fasted for at least $8 \mathrm{~h}$, whilst on the second occasion, measurements were made after each subject had eaten a substantial meal sufficient to produce a feeling of satiation.

Mean postprandial unpotentiated and potentiated $P \mathrm{di}$, tw were significantly greater than corresponding fasting $P_{\mathrm{di}}$,tw in all seven volunteers $(29.8$ versus $25.7 \mathrm{cmH}$ 38.9 versus $34.4 \mathrm{cmH}_{2} \mathrm{O}$, respectively). This was due to a significantly increased gastric pressure component (1.10 versus 0.84 and 0.94 versus 0.78 , respectively), and reduced abdominal compliance $\left(36\right.$ versus $\left.62 \mathrm{~mL} \cdot \mathrm{cmH}_{2} \mathrm{O}^{-1}\right)$. Twitch oesophageal pressure was preserved (15.0 versus $\left.15.4 \mathrm{cmH}_{2} \mathrm{O}\right)$.

The postprandial state increases twitch transdiaphragmatic pressure, and this should be taken into account when using twitch transdiaphragmatic pressure to follow-up patients or to assess the effects of interventions on diaphragm contractility.

Eur Respir J 2002; 20: 577-580.
*Respiratory Muscle Laboratory, Dept of Respiratory Medicine and Allergy, Guy's, King's and St Thomas' School of Medicine, and " Royal Brompton Hospital, London, UK

Correspondence: W. Man, Respiratory Muscle Laboratory, Dept of Respiratory Medicine, King's College Hospital, Bessemer Road, London SE5 9PJ UK.

Fax: 442073463589

E-mail: William.man@kcl.ac.uk

Keywords: Magnetic stimulation phrenic nerves

transdiaphragmatic pressure

Received: December 132001

Accepted after revision: March 42002

W. Man is a Clinical Research Training Fellow of the Medical Research Council (UK).
The diaphragm is innervated exclusively by the phrenic nerves, and thus maximum phrenic nerve stimulation with measurement of twitch transdiaphragmatic pressure $(P \mathrm{di}, \mathrm{tw})$ provides a specific nonvolitional means of investigating the force generating capacity of the muscle. Magnetic stimulation of the phrenic nerves is a painless, reliable method of maximally stimulating the phrenic nerves [1], and is increasingly important as a clinical tool to assess diaphragm strength in patients with neuromuscular disease, neonates and critically ill children and adults. In addition, $P$ di,tw is used to assess the effect of interventions, for example lung volume-reduction surgery, on diaphragm contractility [2], and has been identified by the National Heart Lung and Blood Institute as one of the most promising techniques in the determination of respiratory muscle fatigue [3]. Relatively small reductions in $P$ di,tw have been used to define diaphragm fatigue, requiring precise, reproducible measurements.

However, the relationship between pressure and force is complex. For example, thoraco-abdominal configuration and the mechanical characteristics of the ribcage and abdominal wall with which respiratory muscles interact, play a major role in the efficiency of the conversion of force into pressure $[4,5]$. The effects of abdominal binding, lung volume and posture on $P$ di,tw are well known $[6,7]$, but it is not known whether gastric distension (the consequence of eating a large meal) has an effect on $P$ di,tw. The present authors hypothesised that a substantial meal, sufficient to produce gastric distension, should increase $P$ di,tw through decreased abdominal compliance $(C \mathrm{ab})$. This could be a factor contributing to variability of between-occasion $P$ di,tw. To test this hypothesis, $P$ di,tw, the relative gastric pressure contribution, and $C \mathrm{ab}$ when fasted and postprandial were compared.

\section{Materials and methods}

\section{Study subjects}

Seven healthy volunteers (five males and two females, aged 27-38 yrs), with no history of neuromuscular or respiratory disease, participated in the study. All subjects gave their informed consent, and the local research ethics committee of King's College Hospital approved the study.

\section{Study design}

$P$ di,tw, potentiated and unpotentiated, were measured using bilateral antero-lateral magnetic stimulation of the phrenic nerves (BAMPS) in each subject on two occasions. On the first occasion, the subjects had fasted for at least $8 \mathrm{~h}$. On the second occasion, 
measurements were made after each subject had eaten sufficient slices of pizza to produce a feeling of satiation, and a degree of uncomfortable abdominal bloating sufficient to score at least 3 (moderate) on the modified Borg Scale, which ranges from 0 (none at all) to 10 (maximal). Resting gastric pressures $(P$ ga $)$, gastric pressure component (the ratio between change in $P$ ga to change in oesophageal pressure $\left(P_{\text {oes }}\right)$ during each twitch stimulation: $\left.P_{\text {ga,tw: }} P_{\text {oes,tw }}\right)$ and $C$ ab, calculated as change in abdominal volume displacement over change in abdominal pressure, were also measured. To assess between-occasion lung volume changes, functional residual capacity (FRC) was measured using the helium dilution technique (Jaeger Masterscreen PFT; Jaeger, Hoechberg, Germany), and constancy of end-expiratory lung volume was assessed from the pretwitch resting $P_{\text {oes }}$ trace.

\section{Measurement of twitch transdiaphragmatic pressure}

$P$ di was recorded by measuring $P_{\text {oes }}$ and $P$ ga with a pair of conventionally placed polyethylene balloon catheters (Ackrad Laboratories, Inc., Cranford, NJ, USA). Pressures were measured by differential pressure transducers (Validyne MP45; Validyne, Northridge, CA, USA). The signals from transducers were connected to an analogue-digital board (NB-MIO-16; National Instruments, Austin, TX, USA), and were recorded by a MacIntosh Centis 650 computer using Labview 2.2 software (National Instruments, Austin, TX, USA). Resting $P$ oes and $P$ ga were recorded during quiet breathing to give baseline values. $P$ oes,tw, $P$ ga,tw and $P$ di,tw (the digital subtraction of $P$ oes from $P$ ga) were recorded during each magnetic stimulation, and analysed off-line.

\section{Bilateral antero-lateral magnetic stimulation of the phrenic nerves}

BAMPS was performed, as previously described [8], using two 43-mm figure-of-eight coils powered by Magstim 200 stimulators (Magstim Company Limited, Whitland, Dyfed, Wales) that were linked so they would discharge simultaneously. BAMPS was chosen rather than cervical magnetic stimulation, because this is more likely to be supramaximal than the cervical approach [8], and leads to less contribution from ribcage muscles. The coils were placed on each side of the neck at the posterior border of the sternomastoid muscle at the level of the cricoid cartilage. The subjects were seated comfortably in a chair in an upright posture. One hundred per cent magnetic power output was applied to ensure maximal stimulation of the phrenic nerves [8]. In three subjects (subjects 5-7), supramaximality of $P$ di,tw was confirmed by recording $P$ di,tw at a range of percentages of maximal magnetic power outputs. At least five stimulations were made on each occasion at FRC with the mouth closed, a noseclip in place and belts loosened. To avoid twitch potentiation of the diaphragm there was a rest period of at least $20 \mathrm{~min}$ of quiet breathing before the start of stimulation, and a 1/2-min interval between each twitch $[1,9]$. In addition, $P$ di,tw was measured after deliberately potentiating the diaphragm with a maximum inspiratory manoeuvre $(P \mathrm{I}, \max )$ sustained for $5 \mathrm{~s}[9]$.

\section{Abdominal compliance}

$C$ ab was recorded in the fasting and postprandial state by relating the abdominal wall volume displacement, measured by calibrated inductance plethysmography (Respitrace; Ambulatory Monitoring, Ardsley, NY, USA), with changes in $P_{\text {ga }}$ during tidal breathing.

\section{Data analysis}

Analysis was performed off-line. $P$ di was calculated by digital subtraction of $P$ oes from $P$ ga. Zero $P$ di was taken as the $P$ di at the end of quiet expiration at FRC. Twitch responses were rejected for analysis when $P$ oes immediately before stimulation was $>1 \mathrm{cmH}_{2} \mathrm{O}$ different from that of baseline, and when stimulations were performed during swallowing or oesophageal peristalsis. Fasting and postprandial values were compared using paired t-tests.

\section{Results}

The mean \pm sD data for $P$ di,tw, $P$ oes,tw and $P$ ga,tw are presented in table 1 . The potentiated $P$ di,tw was significantly higher than the unpotentiated twitch $(\mathrm{p}<0.01)$. In all seven subjects, both potentiated and unpotentiated postprandial $P$ di,tw was greater than fasting $P$ di,tw $(\mathrm{p}<0.01)$; the mean increase was $16.3 \%$ (range $2.7-33.2 \%$ ) for unpotentiated $P$ di,tw, and $13.1 \%$ (range $6.6-18.8 \%$ ) for potentiated $P$ di,tw. Postprandial $P$ ga,tw was significantly higher than fasting values (16.4 versus $12.2 \mathrm{cmH}_{2} \mathrm{O}, \mathrm{p}<0.01$ ), whilst $P$ oes, tw was relatively preserved (15.4 versus $\left.15.0 \mathrm{cmH}_{2} \mathrm{O}, \mathrm{p}=0.58\right)$. As a result, there was an increase in the postprandial gastric pressure component (unpotentiated $P$ ga,tw: Poes,tw 1.10 versus $0.84, \mathrm{p}<0.01$; potentiated Pga,tw: $P$ oes,tw 0.94 versus $0.78, \mathrm{p}<0.05)$, illustrated in figure 1 . Additionally, there was an increase in resting $P$ ga $(17.3$ versus $\left.10.7 \mathrm{cmH}_{2} \mathrm{O}, \mathrm{p}<0.01\right)$, and reduced $C \mathrm{ab}(36$ versus $\left.62 \mathrm{~mL} \cdot \mathrm{cm}^{-1} \mathrm{H}_{2} \mathrm{O}, \mathrm{p}<0.02\right)$. No difference was found between fasting and postprandial FRC (3.02 versus $3.00 \mathrm{~L}$ respectively, $\mathrm{p}=0.43$ ) or between mean resting $P_{\text {oes }}\left(-4.0\right.$ versus $\left.-3.7 \mathrm{cmH}_{2} \mathrm{O}, \mathrm{p}=0.15\right)$. Supramaximality of BAMPS was confirmed by a levelling off of $P$ di,tw.

\section{Discussion}

The principal finding of this study is that $P$ di,tw significantly increases following a substantial meal; this is accompanied by a greater contribution from the gastric pressure component, and significantly reduced $C$ ab. Although the amount of food consumed was not controlled (principally because the authors wished to 


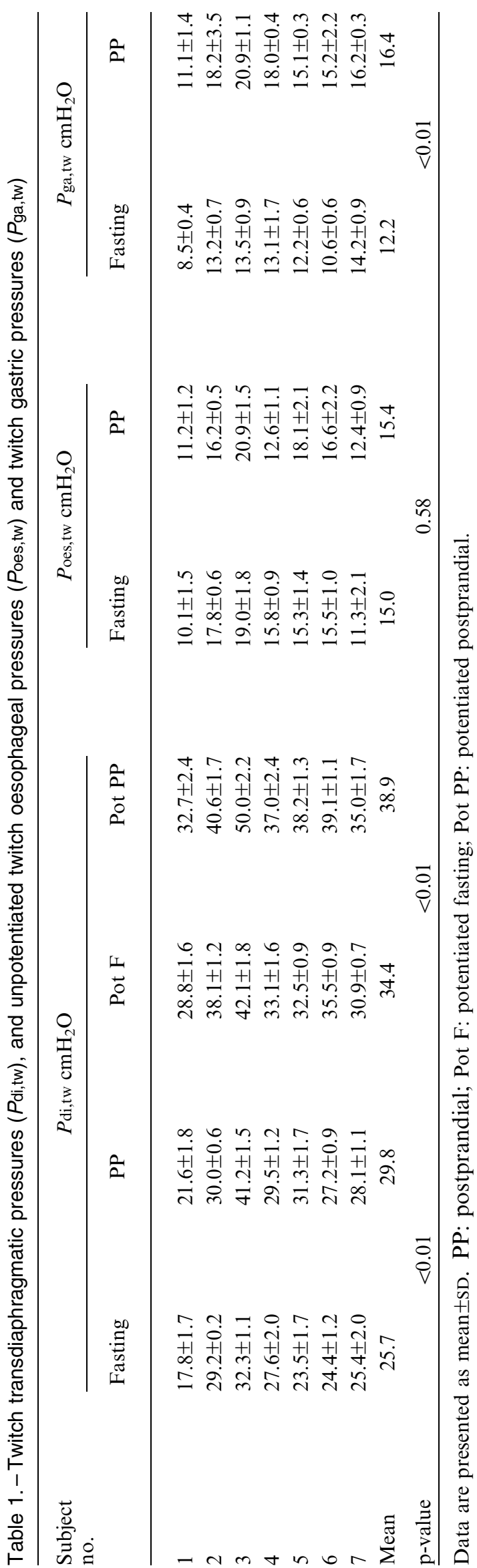

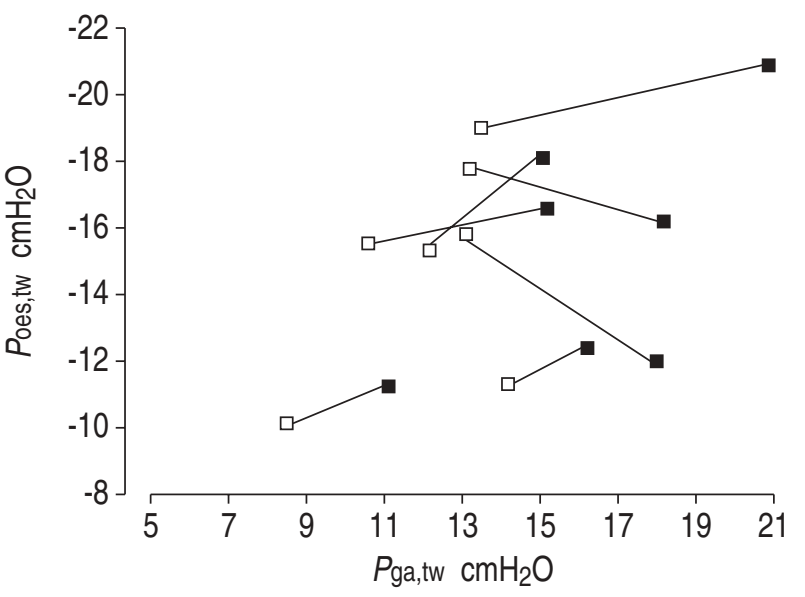

Fig. 1. - Postprandial effects on unpotentiated twitch gastric pressure $\left(P\right.$ ga,tw)/twitch oesophageal pressure $\left(P_{\text {oes,tw }}\right)$ ratio. $\square$ : fasting; 口: postprandial. A right shift of the scatter indicates increasing gastric pressure component contribution following a substantial meal.

simulate real life circumstances, but also the amount of food required to satisfy hunger varied as much as four-fold between subjects), it is interesting to note that subject 2, who showed little change in $P$ di,tw, ate the smallest amount and reported the least amount of abdominal bloating.

Several factors need to be taken into account in the measurement of $P$ di,tw, including potentiation and ensuring supramaximality. In this study, potentiation was avoided by resting subjects for at least $20 \mathrm{~min}$ before stimulation, and allowing 1/2-min intervals between each twitch [9]. Furthermore, a large meal had the same effect on potentiated as well as unpotentiated Pdi,tw. Throughout the study, 100\% magnetic power was used to record $P \mathrm{di}, \mathrm{tw}$, which is more than sufficient to ensure supramaximality [8].

A possible explanation for the changes seen in the postprandial state is a change in lung volume, altering the length/tension properties of the diaphragm. HAMnEGard et al. [6] demonstrated that the unpotentiated $P$ di,tw increases in a linear fashion with decreasing lung volume. Intuitively, one would not expect a marked decrease in lung volume with gastric distension, and this was confirmed by the unchanged FRC and resting $P$ oes in the present study. The method of measuring $C \mathrm{ab}$ in this study was approximate, as it did not take into account vertical volume displacements of the abdomen, but the pelvis prevents caudal movements, whilst the unchanged FRC suggests that cranial movements are unlikely to be significant. Work investigating the compartmental volume changes with acute abdominal distension concluded that the interaction of the ribcage, abdomen, and diaphragm is such that they defend against large changes in end-expiratory lung volume [10].

Previous studies investigating the effect of abdominal binding have shown a marked increase in $P$ di,tw in both humans and animals. In seated healthy subjects, a tight fitting abdominal corset increased $P$ di,tw by $>10 \mathrm{cmH}_{2} \mathrm{O}$ [7]. These changes were principally due to markedly increased intrabdominal 
pressures. By binding the abdomen during phrenic nerve stimulation, the contraction is made closer to isometric than when the abdominal wall is compliant. In experiments involving acute abdominal distension, there is an increase in the gastric pressure component [11], and reduced $C \mathrm{ab}$ [10], consistent with the findings in this study.

The transformation of force into pressure is dependent on many factors, including thoracoabdominal configuration and the mechanical characteristics of the ribcage and abdominal wall $[4,5]$. After a meal, the increasing abdominal pressure may increase the area of apposition of the diaphragm, and increase the hydrostatic pressure applied to the ribcage. Alternatively, the increasing tension of the abdominal wall muscles could pull downward on the lower ribcage.

In this study, gastric distension principally increased $P$ di,tw through an increase in $P$ ga,tw, whilst $P_{\text {oes,tw }}$ was relatively preserved. This raises the possibility of measuring $P_{\text {oes, tw }}$ alone in assessing the inspiratory action of the diaphragm. This would only require the passing of a single balloon catheter, and would be less likely to be affected by confounding factors, including the degree of gastric filling. Several studies have looked at noninvasive, nonvolitional methods of assessing diaphragm strength, such as twitch mouth pressures $(P \mathrm{~m}, \mathrm{tw})$, and the present authors have previously demonstrated a strong correlation between

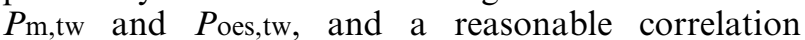
between $P \mathrm{~m}, \mathrm{tw}$ and $P$ di,tw [12]. However, when assessing diaphragm contractility, one has to consider the dual action of the diaphragm: an increase in abdominal pressure, displacing the abdomen outward as well as a lowering of pleural pressure. The abdomen acts as a load upon which the diaphragm exerts its force. Furthermore $P$ di,tw remains the standard means of measuring the effects of fatiguing tasks on diaphragm contractility [3].

The measurement of twitch transdiaphragmatic pressure is an important clinical tool in the investigation of diaphragm strength and contractility, particularly in the assessment and follow-up of patients with neuromuscular disease. Bilateral antero-lateral magnetic stimulation of the phrenic nerves has many advantages over transcutaneous electrical stimulation; it is relatively painless, more reliable in ensuring supramaximal stimulation of the phrenic nerves, and has significantly less within-occasion variability [8]. However, like all biological measurements, there is a degree of between-occasion variability, and in the author's laboratory twitch transdiaphragmatic pressure has a mean coefficient of variation of $11 \%$. This is particularly important when using twitch transdiaphragmatic pressure to detect low-frequency fatigue, with some investigators defining fatigue as a drop of twitch pressure of $\geqslant 10 \%$ [13]. The present authors routinely control for posture, abdominal binding, and potentiation when measuring twitch transdiaphragmatic pressure. However, a meal can substantially increase twitch transdiaphragmatic pressure, and the prandial state of a subject should be taken into account when following-up patients or assessing the effects of interventions on diaphragm contractility.

\section{References}

1. Similowski T, Fleury B, Launois S, Cathala HP, Bouche P, Derenne JP. Cervical magnetic stimulation: a new painless method for bilateral phrenic nerve stimulation in conscious humans. J Appl Physiol 1989; 67: 1311-1318.

2. Laghi F, Jubran A, Topeli A, et al. Effect of lung volume reduction surgery on neuromechanical coupling of the diaphragm. Am J Respir Crit Care Med 1998; 157: 475-483.

3. NHLBI workshop. Respiratory muscle fatigue. $\mathrm{Am}$ Rev Respir Dis 1990; 142: 474480.

4. Chen R, Kayser B, Yan S, Macklem PT. Twitch transdiaphragmatic pressure depends critically on thoracoabdominal configuration. J Appl Physiol 2000; 88: 54-60.

5. Chihara K, Kenyon CM, Macklem PT. Human rib cage distortability. J Appl Physiol 1996; 81: 437-447.

6. Hamnegard CH, Wragg S, Mills G, et al. The effect of lung volume on transdiaphragmatic pressure. Eur Respir J 1995; 8: 1532-1536.

7. Koulouris N, Mulvey DA, Laroche CM, Goldstone J, Moxham J, Green M. The effect of posture and abdominal binding on respiratory pressures. Eur Respir J 1989; 2: 961-965.

8. Mills GH, Kyroussis D, Hamnegard CH, Polkey MI, Green M, Moxham J. Bilateral magnetic stimulation of the phrenic nerves from an anterolateral approach. Am J Respir Crit Care Med 1996; 154: 1099-1105.

9. Wragg S, Hamnegard C, Road J, et al. Potentiation of diaphragmatic twitch after voluntary contraction in normal subjects. Thorax 1994; 49: 1234-1237.

10. Gilroy RJ Jr, Lavietes MH, Loring SH, Mangura BT, Mead J. Respiratory mechanical effects of abdominal distension. J Appl Physiol 1985; 58: 1997-2003.

11. Desmecht D, Linden A, Lekeux P. Pathophysiological response of bovine diaphragm function to gastric distension. J Appl Physiol 1995; 78: 1537-1546.

12. Hamnegard $\mathrm{CH}$, Wragg $\mathrm{S}$, Kyroussis D, et al. Mouth pressure in response to magnetic stimulation of the phrenic nerves. Thorax 1995; 50: 620-624.

13. Mador MJ, Rodis A, Magalang UJ, Ameen K. Comparison of cervical magnetic and transcutaneous phrenic nerve stimulation before and after threshold loading. Am J Respir Crit Care Med 1996; 154: 448453. 